\title{
Lidil
}

Revue de linguistique et de didactique des langues

$58 \mid 2018$

L'enseignement et l'apprentissage de l'écrit académique à l'aide de corpus numériques

\section{Vers une socioterminologie médicale wolofe}

Towards a Wolof Medical Socioterminology

\section{Abibatou Diagne et Abou Bakry Kébé}

\section{OpenEdition}

\section{Journals}

Édition électronique

URL : http://journals.openedition.org/lidil/5029

DOI : $10.4000 /$ lidil.5029

ISSN : 1960-6052

Éditeur

UGA Éditions/Université Grenoble Alpes

Édition imprimée

ISBN : 978-2-37747-064-8

ISSN : 1146-6480

Référence électronique

Abibatou Diagne et Abou Bakry Kébé, «Vers une socioterminologie médicale wolofe », Lidil [En ligne], 58 | 2018, mis en ligne le 02 novembre 2018, consulté le 30 avril 2019. URL : http:// journals.openedition.org/lidil/5029; DOI : 10.4000/lidil.5029

Ce document a été généré automatiquement le 30 avril 2019

(C) Lidil 


\section{Vers une socioterminologie médicale wolofe}

Towards a Wolof Medical Socioterminology

Abibatou Diagne et Abou Bakry Kébé

\section{De la socioterminologie}

1 Dans son ouvrage, Gaudin (2003) se démarque de l'approche fixiste wüsterienne de la terminologie. En effet, guidé par une logique et une visée essentiellement normalisatrices, Wüster a conçu une théorie terminologique axée sur une relation biunivoque entre terme et concept. En introduisant une dimension sociale, Gaudin confère à la terminologie un caractère mouvant avec «la référence conçue comme un acte dynamique de co-construction perpétuelle du monde »(Dufour, 2004, p. 208).

2 Adopter une approche socioterminologique implique de prendre en compte que la monosémie du terme n'exclut pas que celui-ci puisse appartenir à plusieurs registres de discours. Dès lors, une communauté est en mesure de se le réapproprier. La recherche et la constitution de termes en contexte plurilingue sont d'une certaine manière influencées par le contact des langues et tiennent nécessairement compte de variables sociales telles que les rapports qu'entretiennent les locuteurs avec leur langue. Gaudin (2003) identifie également la vulgarisation scientifique (VS) comme un moyen de diffusion de savoirs.

Pour occuper de nouveaux espaces de parole, une langue se renouvelle, dans le sens d'un enrichissement de son lexique. Cela passe par l'appropriation de concepts, relativement nouveaux ou qui se renouvellent chaque fois dans les domaines techniques ou scientifiques. Notre approche socioterminologique consiste en une observation de l'usage d'emprunts chez les locuteurs dans le domaine médical, puis en une proposition de termes substituables aux emprunts et enfin en une détermination de facteurs d'implantation. 


\section{Situation sociolinguistique : contact des langues}

Les situations de contact de langues constituent des facteurs importants de changements linguistiques. Une langue peut s'enrichir grâce à son pouvoir d'assimilation, à sa plus ou moins grande perméabilité ou encore au processus de normaison ${ }^{1}$ des locuteurs et/ou de normalisation ${ }^{2}$ des instances officielles. "L'intervention autoritaire et prestigieuse " (Glissant, 1981) du français et des autres langues étrangères s'est faite au détriment des langues du terroir en Afrique.

5 Le contact donne lieu à un processus d'interpénétration aux enjeux linguistiques multiples qui dépendent du type de rapport établi. Le degré d'interpénétration peut ainsi être très élevé lorsqu'il y a une proximité géographique et linguistique. Le contact entre langues est partiellement lié à la proximité géographique. Ainsi, l'enclave gambienne du Sénégal utilise comme principale langue véhiculaire le wolof ; la Mauritanie, zone tampon entre le Maghreb et l'Afrique noire, est aussi un creuset culturel où se brassent plusieurs langues.

6 Toutefois, les rapprochements physique ou linguistique ne sont pas les seules causes de contact. Il peut aussi être le fait de l'histoire, d'une idéologie, du développement culturel ou économique. Ces types de contact ont la caractéristique de profondément remettre en cause des modèles établis et de se poser en une sorte d'alternative nouvelle et attractive. L'école sénégalaise n'est conçue dans sa forme classique que presque exclusivement à travers l'apprentissage du français et, dans une moindre mesure, des autres langues étrangères - ce qui relègue les langues nationales du pays à "des fonctions basses » (Fishman, 1967). La connaissance de la religion musulmane est indissociable d'une connaissance, ne serait-ce que passive, de l'arabe, alors que l'acquisition de compétences en anglais s'est érigée en nécessité pour une jeunesse de plus en plus influencée par la culture anglo-saxonne.

7 En contexte plurilingue sénégalais, le contact des langues nationales a eu pour conséquence un effacement progressif du paramètre «langue» comme critère d'appartenance ethnique. En effet, du fait du caractère quelque peu assimilateur du wolof, l'on constate une forte disparité entre locuteurs (langue parlée par $80 \%$ de la population selon Mbodj, 1994) et membres de l'ethnie wolofe. Par ailleurs, les différents parlers de cette langue ou encore ses variations régionales n'ont jamais constitué un élément de blocage quant à l'intercompréhension entre locuteurs et n'instaurent donc pas une barrière entre wolof du Jolof, du Waalo ou encore le lébou considéré comme le véritable parler source du wolof tel qu'il se présente aujourd'hui (Robert, 1991).

8 En revanche, l'opposition wolof urbain/wolof rural peut être considérée comme une des conséquences de l'ouverture de cette langue à la modernité ; ou encore à sa cohabitation avec le français et les langues étrangères. Le wolof rural représente cet idéal de wolof «pur » ou de la langue dans sa version la moins emprunteuse. Le wolof parlé en milieu urbain est celui des jeunes qui lui impulsent son dynamisme créateur.

\section{3. Éléments comparatifs de concepts}

9 La richesse terminologique d'une langue, dans un domaine donné, est fonction du niveau de développement de la culture scientifique dudit domaine dans cette langue. Le 
développement des langues de spécialité - qui véhiculent par la même occasion des cultures scientifiques - remet en cause l'une des caractéristiques de base de la langue qui est d'être un élément porteur d'un angle de vue interne qui proviendrait du vécu et des pratiques d'une communauté donnée. Par le truchement de l'emprunt, ce ne sont pas que des items ou encore des expressions qui passent d'une langue à une autre, c'est aussi tout un vécu de pratiques sociales, culturelles, langagières, des modes de pensées.

Les liens entre langue et culture ont souvent été étudiés selon le rôle véhiculaire joué par la première sur la seconde. Le langage a un rôle expressif qui montre des faits et objets communs aux humains. Toutefois dans le cadre des activités humaines, l'on trouve des besoins d'expression différents. Ainsi, si la santé intéresse tout individu, les pratiques médicales restent un domaine réservé en principe aux spécialistes. Les méthodes peuvent différer, mais la nouveauté comme élément qui justifierait l'emprunt à une autre langue ou l'inexistence dans la langue emprunteuse ne sont que trop souvent invoqués, dans le cas d'une langue comme le wolof.

11 Le concept dans une approche purement linguistique est considéré comme un équivalent du signifié (Gaudin, 2003, p.61). Sa dimension extralinguistique qui découle d'une abstraction se trouve fortement défendue par Cabré (1998) à la suite de l'ISO ${ }^{3}$ :

Les concepts, ou représentations mentales des objets sont le fruit du choix des caractères pertinents qui définissent une classe d'objets et non pas des objets individuels. (p. 168)

12 Sur un plan philosophique, Gaudin (2003) ne manque pas de souligner le caractère actif de la construction du concept qui consiste moins à restituer le monde des choses qu'à le « redessiner en lui substituant un ordre d'un autre type » (p. 62). Les exemples de DikiKidiri (1999) pour désigner la bicyclette sont très parlants :

- gbâzâbängâ, « roues de caoutchouc », en sängö (Centrafrique) ;

- nàgàsó, « cheval de fer ", en bambara (Mali);

- magu-mâkwanganya, "quatre pieds", en lilikô (langue bantu de la zone D en

République démocratique du Congo). (p. 579)

13 Le sangö redessine la bicyclette en désignant une partie pour le tout, procédé métonymique qui interroge dans une perspective socioterminologique et qui suggèrerait l'existence de roues faites essentiellement dans une autre matière. Diki-Kidiri rappelle que les Centrafricains étaient soumis aux travaux forcés de la récolte du caoutchouc végétal durant la colonisation. De cet artéfact, ils retiennent l'élément qui leur rappelle une expérience qu'ils ont eue en partage. Cette représentation métonymique en rappelle une autre qu'on retrouve pour désigner le train en wolof (saxaar, "fumée»). Une désignation qui fait écho aux nuages de fumée que dégageaient les premiers trains et qui subsiste sur les panneaux routiers indiquant les passages à niveau un peu partout dans le monde.

14 La langue bambara redessine la bicyclette en référence à un animal, mais aussi au fer qui apporte un programme de sens de la modernité, alors que le lilikô véhicule l'image d'une personne utilisant un véhicule qui «dédouble ses pieds» et se retrouve avec «quatre pieds ». Dans tous ces cas de figure, l'on a affaire à une praxis expérientielle qui, loin de souligner les traits saillants de l'artéfact de départ, met en avant les aspects sociodiscursifs et historiques de la langue et les praxis sociales et culturelles.

Les recherches terminologiques en wolof sont davantage axées sur le domaine médical, notamment avec les travaux de Faal (1994, 1995), Faal et Njaay (1994a, 1994b, 1994c), Mbodj (2014) et Diagne (2018). Chez les deux premiers auteurs, ainsi que chez d'autres 
auteurs ayant travaillé avec le $\mathrm{CLAD}^{4}$, l'on note une approche qui laisse une place à la vulgarisation et soulève des questions sociodiscursives pertinentes. Dans ces livrets de vulgarisation, il est fréquent de trouver des dialogues typiques des échanges médecinspatients, ce qui contextualise socialement une pratique spécialisée.

La thématique de la santé dans le cadre culturel wolof peut être divisée en deux sousgroupes : médecine traditionnelle et médecine moderne. Sous le vocable de traditionnel ( paju wolof) sont réunies toutes les pratiques médicales qui relèvent de méthodes du terroir puisées à partir d'approches ancestrales où se mêlent science et syncrétisme. La médecine moderne, quant à elle (paju tubaab), représente l'ensemble des pratiques médicales implantées par les Européens. Cette dernière ne s'est pas affranchie de sa principale langue véhiculaire, malgré son implantation en milieu wolophone: case de santé, centre de santé, hôpital, ministère de la santé, médecin, sage-femme, gynécologue, tuberculose, diabète, etc. C'est donc une catégorie conceptuelle de la santé subdivisée par le simple fait de l'utilisation d'une langue plutôt que d'une autre. Les termes à développer pourraient s'inscrire dans un contexte aménagiste essentiellement axé sur la désignation des affections et des équipements thérapeutiques.

\section{La dénomination des affections médicales en wolof}

Les affections ${ }^{5}$ en wolof sont désignées de manière générique selon les régions ou parties du corps affectées :

(1) Feebaru tànk (glose : Maladie+de pied)

$>$ maladies qui affectent les membres inférieurs

(2) Feebaru bët (glose : Maladie+de yeux)

$>$ maladies qui affectent les yeux

(3) Feebaru xol (glose : Maladie+de cœur)

$>$ maladies cardiovasculaires

(4) Feebaru xel (glose : Maladie+de esprit)

$>$ maladies mentales

Suivant une logique de conceptualisation qui se fonde sur les conséquences des maladies, il y a une subdivision en deux grandes catégories :

(5) Feebar buy wàllate (glose : Maladie Relat+Inacc contamine)

$>$ maladies contagieuses

(6) Feebar bu dul wàllate (glose : Maladie Relat NEG contamine)

> maladies non contagieuses

La troisième grande catégorie est dénommée sur la base des causes, des origines des maladies :

(7) Feebaru ndono (glose : Maladie+de hérédité)

> maladies génétiques ou héréditaires

L'on pourrait donc dire en wolof, dans le même ordre d'idée :

(8) Feebaru koromosome (glose : maladie+de chromosome)

$>$ maladies chromosomiques

(9) Feebar bu tukke ci doomu jàngoro (glose: maladie Relat provient de micro organisme)

$>$ maladies microbiennes et virales

(10) Feebar bu tukke ci xeetu saan (glose: maladies Relat provient de semblables parasites)

> maladies parasitaires 
(11) Feebar bu tukke ci lu bare (glose : maladies Relat provient de Relat varié)

$>$ maladies multifactorielles

(12) Feebar bu tukke ci ñàkk (glose : maladie Relat provient de carence)

$>$ maladies dues aux carences

(13) Feebaru xel mu dal ak yaram (glose: Maladie+de esprit Relat tranquille et corps)

> maladies psychosomatiques et maladies psychiques

L'élaboration de terminologies wolofes dans un cadre scientifique n'est pas encore complètement aboutie. Il faut surtout relever des travaux à caractère quelque peu artisanal, mais aussi fortement orientés vers la vulgarisation.

\section{5. Élaboration de terminologies en wolof, perspective vulgarisatrice}

Les recherches en terminologie s'articulent depuis plus de deux décennies autour d'une prise en compte du cadre culturel, comme le défendent les membres de l'école de Rouen à travers la socioterminologie.

Par ailleurs, d'après nos observations sur la langue médicale wolofe, la désignation est la seule voie qui permet de trouver des équivalents qui peuvent se substituer à la multitude de termes empruntés au français. Comme le souligne Gaudin (2003), désigner nécessite une connaissance parfaite de l'objet ou de la notion afin de mettre en avant les traits principaux qui apparaissent dans cette désignation :

[...] la désignation, la construction de la référence, sont des questions relevant de la mise en action de la langue dans une interlocution. Elles relèvent d'une pragmatique socialisée et historicisée [...]. (p. 33)

Le référent apparait comme la représentation physique et le concept comme la représentation mentale, mais les deux concourent au même but en terminologie. La conceptualisation demeure un élément plutôt ancré dans l'objectivité et elle doit être dissociée, comme le souligne Diki-Kidiri (1999), du percept purement subjectif et dépendant du vécu, des pratiques, de l'interaction avec les éléments qui se situent dans l'environnement d'une communauté donnée.

La notion de partenariat linguistique telle que défendue dans certaines instances glottopolitiques comme l'OIF $^{6}$, avec les valeurs de partage et d'affirmation d'identités spécifiques, est très utile dans l'appréhension des sciences et techniques des langues nationales. En effet, le Sénégal est un espace plurilingue et francophone qui, dans le cadre de sa politique terminologique (qui reste à définir), gagnerait à donner une place au français qui ne serait pas qu'une langue d'emprunts, mais aussi - parce que terminologiquement mieux dotée et sociolinguistiquement proche du wolof - une langue de référence, de conceptualisation au plus près des réalités du milieu. Il reste que la politique linguistique du Sénégal s'inscrit également dans une politique culturelle construite qui situe les langues au cœur des processus d'expression de la culture sénégalaise à travers les arts, la littérature, etc.

Les différents écrits médicaux en wolof sont orientés vers la vulgarisation, comme on peut le noter chez Faal et Njaay (1994a, 1994b, 1994c), Faal (1995), INEPES (2006), Mbodj (2014). Cela témoigne de la dimension sociale que représente la constitution de termes chez ces auteurs et il parait utile de la prendre en compte. 
La vulgarisation s'articule autour de deux axes : informer et assurer la diffusion de savoirs à grande échelle; elle constitue également sur le plan linguistique un travail de substitution qui laisse une place à la rhétorique. Dans nos travaux antérieurs (Diagne, 2018, 2015), l'accent a été mis, au plan linguistique, sur le caractère expansif des processus de désignation indissociables de la démarche vulgarisatrice. Par ailleurs, le wolof, comme beaucoup de langues négro-africaines, a une caractéristique verbalisante qui incite à avoir recours à des séquences expansives (Diagne, 2018) :

Tuberculose : Sëqët su bon si

Angiocholite : Mettitu waruwaa yi jëm ci wextan

Agraphie : Jafe jafe mën bind

Le caractère peu doté, c'est-à-dire l'absence ou la rareté de terminologies, appelle une technique propre à la vulgarisation : la reformulation. L'implantation des termes wolofs, à notre sens, passe nécessairement par le canal de la vulgarisation. En effet, celle-ci confère une notion utilitaire à la pratique terminologique dans l'aire culturelle du wolof tout en renforçant les pouvoirs d'expression de cette langue. L'idée majeure est bien sûr d'élaborer des termes, mais également d'arriver à une conception et une expression des sciences médicales au plus près des réalités socioculturelles wolofes.

\section{Conclusion}

Nous avons étudié les modalités de dénomination/désignation dans la terminologie médicale d'une langue peu aménagée, dans un espace sociolinguistique sénégalais marqué par le contact des langues. En effet, la vitalité des langues du Sénégal, du wolof en particulier, s'est traduite, depuis quelques années, par des besoins pressants en termes de lexique spécialisé. À travers quelques exemples pris dans le champ médical, nous avons vu comment les liens référentiels peuvent contribuer à rendre moins opaque la terminologie. Aujourd'hui, l'accès à une information médicale compréhensible est devenu un enjeu majeur de santé publique dans tous les pays et il y a un besoin de structuration légitime de la terminologie médicale au Sénégal. Mener des recherches terminologiques dans le contexte socioculturel sénégalais passe par une mise «en place des structures chargées de la standardisation des variétés des langues retenues, de leur normalisation et de leur enrichissement conceptuel » (Cissé, 2005, p. 128). Cela relève fondamentalement d'une volonté politique.

Le statut du wolof comme langue la plus parlée au Sénégal et son importance dans les interactions médicales peuvent justifier une intervention terminologique sur cette langue. Mais le succès d'un tel aménagement linguistique/terminologique dépendra de la capacité de l'État à ne pas accréditer une vision "glottophage » du wolof au Sénégal, aux dépens de la variation et du plurilinguisme (Juillard \& Dreyfus, 2005, p. 320).

BIBLIOGRAPHIE

CABRÉ, Maria Theresa. (1998). La terminologie théorie, méthodes et applications. Paris : Armand Colin. 
CISSÉ, Mamadou. (2005). Langues, État et société au Sénégal. Sudlangues, 5, 99-133.

DiAGNE, Abibatou. (2014). De quelques problèmes de traduction des adjectifs relationnels du français vers le wolof : étude sur corpus de terminologie commerciale. Dans M. Mangeot \& F. Sadat (dir.), Actes de l'atelier sur le traitement automatique des langues africaines - TALAf 2014. Disponible en ligne sur <http://talaf.imag.fr/2014/> (consulté le 23 septembre 2018).

DiAGNE, Abibatou. (2018). La terminologie wolof dans une perspective de traduction et de combinatoire lexicale restreinte (Thèse de doctorat). Université Lumière - Lyon 2, Lyon.

DiKI-KIDIRI, Marcel. (1999). Le signifié et le concept dans la dénomination. Meta: Journal des traducteurs / Meta: Translators' Journal, 44(4), 573-581.

DuFour, Françoise. (2004). François Gaudin, Socioterminologie, une approche sociolinguistique de la terminologie. Cahiers de praxématique, 42, 208-212.

FAAL, Aram. (1994). Ëmbu jàmm, la maternité. Dakar : OSAD.

FAAL, Aram. (1995). Éducation pour la santé. Dakar : OSAD.

FAAL, Aram \& NJAAY, Mamadu D. (1994a). Sama doom dama koy nàmpal. Dakar : OSAD.

FAAL, Aram \& NJAAY, Mamadu D. (1994b). Lekkug tànneef. Dakar : OSAD.

FAAL, Aram \& NJAAY, Mamadu D. (1994c). Programme élargi de vaccination (PEV). Dakar : OSAD.

Fischman, Joshua A. (1967). Bilingualism with and without Diglossia; Diglossia with and without Bilingualism. Problems of Bilingualism, Journal of Social issues, 23(2), 29-38.

GAUDIN, François. (2003). Socioterminologie : une approche sociolinguistique de la terminologie. Bruxelles : De Boeck \& Larcier / Duculot.

GAUDIN, François. (2005). La socioterminologie. Langages, 157, 80-92.

GLISSANT, Édouard. (1981). Le discours antillais. Paris : Seuil.

INEPES. (2006). Teere wer gu yaram, livret de santé bilingue. Paris : INEPES.

JuILLARD, Caroline \& DREYFus, Martine. (2005). Le plurilinguisme au Sénégal : langues et identités en devenir. Paris : Éditions Karthala.

МворJ, Chérif. (1994). L’activité terminologique au Sénégal. RINT, 11, 3-8.

MворJ, Chérif. (2014). Pour une terminologie de la santé en wolof. Dakar : Presses universitaires de Dakar.

NDAO, Papa Alioune \& KÉBÉ Abou Bakry. (2010). Langues et médias au Sénégal : une expérience de normalisation langagière par les journalistes des radios privées. Enjeux et limites. Dans P. A. Ndao \& A. B. Kébé (dir.), Nouveaux médias et dynamiques des langues dans l'espace francophone, Glottopol, 14, 17-36. Disponible en ligne sur <http://glottopol.univ-rouen.fr/numero_14.htm> (consulté le 20 septembre 2015).

ROBERT, Stéphane. (1991). Approche énonciative du système verbal : le cas du wolof. Paris : Éditions du Centre national de la recherche scientifique.

\section{NOTES}

1. La normaison concerne l'intercompréhension dans les usages langagiers. 
2. La normalisation optimise la communication spécialisée parce qu'elle est prescriptive. Elle constitue un processus par lequel une instance statue sur un terme face à une prolifération de terminologies ou à une absence de terme approprié.

3. L'Organisation internationale de normalisation (de l'anglais International Standard Organisation) établit et publie des normes internationales. La norme ISO 704: 2009 définit et harmonise les principes de base ainsi que les méthodes utilisées dans l'élaboration et la compilation de terminologies.

4. Centre de linguistique appliquée de Dakar, centre de recherche linguistique qui s'intéresse aux questions de traduction, de terminologie et de lexicologie essentiellement, aussi bien pour le français l'anglais que les langues nationales du Sénégal.

5. Les abréviations et symboles utilisés sont les suivantes :

+ : amalgame ou suffixation

NEG : Négatif

Relat : Relatif

Inacc : Inaccompli

6. Cf. le discours de l'ancien secrétaire général de l'OIF, en ligne sur <www.francophonie.org/FesConference-internationale-sur.html> (consulté le 20 octobre 2015).

\section{RÉSUMÉS}

L'usage des langues d'Afrique subsaharienne a connu ces dernières années des changements majeurs aux conséquences sociolinguistiques notables. Dans bien des situations, de nouvelles pratiques communicatives et de nouvelles formes de pouvoirs langagiers sont apparues, en particulier sous l'impulsion de la révolution numérique (Ndao \& Kébé, 2010). Il résulte de ces faits d'importantes reconfigurations $\mathrm{du}$ paysage sociolinguistique. Ainsi, certaines langues connaissent un regain de vitalité et ne sont plus confinées à des «fonctions basses » (Fishman, 1967).

La socioterminologie constitue une branche de la terminologie à dimension sociocritique fortement influencée par la sociolinguistique théorique et la sociolinguistique de terrain (Gaudin, 2005). Elle consiste à faire le lien entre les termes produits et leur contexte d'occurrence. Elle a donc comme objet "l'étude de la circulation des termes en synchronie et en diachronie " (Gaudin, 2005, p. 81). La socioterminologie s'intéresse aux significations socialement réglées des termes. Dans le présent article, nous relevons les conséquences du contact de langues sur les processus de création de lexiques de spécialité en wolof. Nous prenons des exemples du domaine médical qui constitue un terreau fertile à l'usage de termes français en milieu wolophone. En dernière analyse, il conviendra de s'arrêter sur la dimension vulgarisatrice de la terminologie wolofe. L'étude sur corpus d'écrits à caractère médical en wolof montre qu'à l'heure actuelle, la vulgarisation scientifique (VS) constitue le principal canal de diffusion du discours médical et des termes médicaux (Diagne, 2018). La VS se pose en cadre d'appropriation de savoirs, mais aussi en cadre didactique et d'information qui cible les locuteurs wolofs.

In recent years, the use of sub-Saharan African languages has undergone major changes with notable sociolinguistic consequences. In many cases, new communicative practices and new forms of language power have emerged, particularly thanks to digital revolution (Ndao \& Kébé, 2010). These facts result in important reconfigurations of the sociolinguistic landscape and some 
languages are experiencing certain revival of vitality.

Socioterminology is a branch of socio-critical terminology strongly influenced by theoretical sociolinguistics and field sociolinguistics (Gaudin, 2005). It consists in making the link between terms and their context of occurrence. Its object is "the study the terms circulation in synchrony and diachrony" (Gaudin, 2005, p. 81). In this paper, we note the consequences of language contact on the process of terminology creation in Wolof. We take examples from the medical field which is fertile ground for the use of French terms in Wolof area. We insist on the popularizing dimension of Wolof terminology. The study of medical literature in Wolof shows that scientific popularization (SP) is currently the main channel for the diffusion of medical discourse and medical terms (Diagne, 2018). SP is a framework for the appropriation of knowledge, but also a didactic and information framework that targets Wolof speakers.

INDEX

Mots-clés : wolof, socioterminologie, corpus médical

Keywords : Wolof, socioterminology, medical corpus

\section{AUTEURS}

\section{ABIBATOU DIAGNE}

Laboratoire CRTT, Université Lumière - Lyon 2

diagneabi@gmail.com

ABOU BAKRY KÉBÉ

Université Gaston Berger de Saint-Louis (Sénégal)

aboubakry.kebe@gmail.com 